\title{
On Optimal Control Problem for Backward Stochastic Doubly Systems
}

\author{
Adel Chala \\ Laboratory of Applied Mathematics, University Mouhamed Khider, P.O. Box 145, 07000 Biskra, Algeria \\ Correspondence should be addressed to Adel Chala; adel.chala@univ-biskra.dz
}

Received 15 December 2013; Accepted 16 January 2014; Published 4 March 2014

Academic Editors: F. Jauberteau and F. Sartoretto

Copyright ( 2014 Adel Chala. This is an open access article distributed under the Creative Commons Attribution License, which permits unrestricted use, distribution, and reproduction in any medium, provided the original work is properly cited.

We are going to study an approach of optimal control problems where the state equation is a backward doubly stochastic differential equation, and the set of strict (classical) controls need not be convex and the diffusion coefficient and the generator coefficient depend on the terms control. The main result is necessary conditions as well as a sufficient condition for optimality in the form of a relaxed maximum principle.

\section{Introduction}

In 1994, Pardoux and Peng [1] considered a new kind of BSDE, that is a class of backward doubly stochastic differential equations (BDSDEs in short) with two different directions of stochastic integrals, that is, the equations involve both a standard (forward) stochastic Itô integral $d W_{t}$ and a backward stochastic Itô integral $d B_{t}$. More precisely, they dealt with the following BDSDE:

$$
\begin{gathered}
d Y_{t}=f\left(t, Y_{t}, Z_{t}\right) d t+g\left(t, Y_{t}, Z_{t}\right) \overleftarrow{d B}_{t}-Z_{t} d W_{t}, \\
Y_{T}=\xi .
\end{gathered}
$$

They proved that if $f$ and $g$ are uniform Lipschitz, then (1), for any square integrable terminal value $\xi$, has a unique solution $\left(Y_{t}, Z_{t}\right)$ in the interval $[0, T]$. They also showed that BDSDEs can produce a probabilistic representation for solutions to some quasi-linear stochastic partial differential equations. Since this first existence and uniqueness result, many papers have been devoted to existence and/or uniqueness result under weaker assumptions. Among these papers, we can distinguish two different classes: Scalar BDSDEs and multidimensional BDSDEs. In the first case, one can take advantage of the comparison theorem: we refer to Shi et al. [2] who weakened the uniform Lipschitz assumptions to linear growth and continuous conditions by virtue of a comparison theorem introduced by them. They obtained the existence of solutions to BDSDEs but without uniqueness. In this spirit, let us mention the contributions of N'zi and Owo [3], which dealt with discontinuous coefficients. For multidimensional BDSDE, there is no comparison theorem, and to overcome this difficulty, a monotonicity assumption on the generator $f$ in the variable $y$ is used. This appears in the works of Peng and Shi [4] which have introduced a class of forward backward doubly stochastic differential equations, under the Lipschitz condition and monotonicity assumption. Unfortunately, the uniform Lipschitz condition cannot be satisfied in many applications. More recently, N'zi and Owo [5] established existence and uniqueness result under nonLipschitz assumptions. Moreover the authors apply his theory to solve the financial model of cash flow valuation.

In this paper, we study a stochastic control problem where the system is governed by a nonlinear backward doubly stochastic differential equation (BDSDE) of the type

$$
\begin{gathered}
d y_{t}^{\nu}=-f\left(t, y_{t}^{\nu}, z_{t}^{\nu}, v_{t}\right) d t+g\left(t, y_{t}^{\nu}, z_{t}^{\nu}, v_{t}\right) d B_{t}+z_{t}^{v} d W_{t}, \\
y_{T}^{v}=\xi .
\end{gathered}
$$

The control variable $v=\left(v_{t}\right)$, called strict control, is an $\mathscr{F}_{t}$-adapted process with values in some sets $U$ of $\mathbb{R}^{k}$. We denote by $\mathcal{U}$ the class of all strict controls. 
The criteria to be minimized, over the set $\mathcal{U}$, has the following form:

$$
J(\nu)=\mathbb{E}\left[\Psi\left(y_{0}^{v}\right)+\int_{0}^{T} l\left(t, y_{t}^{v}, z_{t}^{v}, v_{t}\right) d t\right],
$$

where $\Psi$ and $l$ are given maps and $\left(y_{t}, z_{t}\right)$ is the trajectory of the system controlled by $\nu$.

A control $u \in \mathcal{U}$ is called optimal if it satisfies

$$
J(u)=\inf _{\nu \in \mathscr{U}} J(\nu)
$$

Our objective in this paper is to establish necessary as well sufficient optimality conditions, of the Pontryagin maximum principle type, for relaxed models.

In this paper, we solve this problem by using an approach developed by Bahlali [6], and it was developed by Chala [7, 8]. We introduce then a bigger new class $\mathscr{R}$ of processes by replacing the $U$-valued process $\left(v_{t}\right)$ by a $\mathbf{P}(U)$-valued process $\left(q_{t}\right)$, where $\mathbf{P}(U)$ is the space of probability measures on $U$ equipped with the topology of stable convergence. This new class of processes is called relaxed controls, and it has a richer structure of convexity, for which the control problem becomes solvable.

The main idea is to use the property of convexity of the set of relaxed controls and treat the problem with the method of convex perturbation on relaxed controls (instead of that of the spike variation on strict one). We establish then necessary and sufficient optimality conditions for relaxed controls.

In the relaxed model, the system is governed by the BDSDE as follows:

$$
\begin{aligned}
d y_{t}^{q}= & -\int_{U} f\left(t, y_{t}^{q}, z_{t}^{q}, a\right) q_{t}(d a) d t \\
& +\int_{U} g\left(t, y_{t}^{q}, z_{t}^{q}, a\right) q_{t}(d a) d B_{t}+z_{t}^{q} d W_{t}, \\
y_{T}^{q}=\xi . &
\end{aligned}
$$

The expected cost to be minimized, the relaxed model, is defined from $\mathscr{R}$ into $\mathbb{R}$ by

$$
\mathscr{J}(q)=\mathbb{E}\left[\Psi\left(y_{0}^{q}\right)+\int_{0}^{T} \int_{U} l\left(t, y_{t}^{q}, z_{t}^{q}, a\right) q_{t}(d a) d t\right] .
$$

A relaxed control $\mu$ is called optimal if it solves

$$
\mathscr{J}(\mu)=\inf _{q \in \mathscr{R}} \mathscr{J}(q) \text {. }
$$

Existence of an optimal solution for this problem has been solved to achieve the objective of this paper and establish necessary and sufficient optimality conditions for these two models, we proceed as follows.

Firstly, we give the optimality conditions for relaxed controls. The idea is to use the fact that the set of relaxed controls is convex. Then, we establish necessary optimality conditions by using the classical way of the convex perturbation method. More precisely, if we denote by $\mu$ an optimal relaxed control, and $q$ is an arbitrary element of $\mathscr{R}$, then with a sufficiently small $\theta>0$ and for each $t \in[0, T]$, we can define a perturbed control as follows $\mu_{t}^{\theta}=\mu_{t}+\theta\left(q_{t}-\mu_{t}\right)$.

By using the fact that the coefficients $f, g$, and $l$ are linear with respect to the relaxed control variable, necessary optimality conditions are obtained directly in the global form.

We note that necessary optimality conditions for relaxed controls, where the systems are governed by a stochastic differential equation, were studied by Mezerdi and Bahlali [9] and Bahlali [6], and we note also that necessary optimality conditions for Stochastic controls, where the systems are governed by forward-backward doubly stochastic differential equation, were studied by Bahlali and Gherbal [10] and Han, Peng, and $\mathrm{Wu}[11]$.

The paper is organized as follows. In Section 2, we give the precise formulation of the problem and introduce the relaxed model. We formulate the problem and give the various assumptions used throughout the paper. In Section 3, we give our first main result, the necessary optimality conditions for control problem and under additional hypothesis. In Section 4, we derive our second main result in this paper, which is sufficient conditions of optimality for relaxed controls.

Along this paper, we denote by $C$ some positive constants and for simplicity, we need the following matrix notations. We denote by $\mathscr{M}_{n \times d}(\mathbb{R})$ the space of $n \times d$ real matrix and $\mathscr{M}_{n \times n}(\mathbb{R})$ the linear space of vectors $M=\left(M_{1}, \ldots, M_{d}\right)$ where $M_{i} \in \mathscr{M}_{n \times n}(\mathbb{R})$. For any $M, N \in \mathscr{M}_{n \times n}^{d}(\mathbb{R}), L, S \in \mathscr{M}_{n \times d}(\mathbb{R})$, $Q \in \mathscr{M}_{n \times d}(\mathbb{R}), \alpha, \beta \in \mathbb{R}^{n}$, and $\gamma \in \mathbb{R}^{d}$, we use the following notations:

$\alpha \beta=\sum_{i=1}^{n} \alpha_{i} \beta_{i}$ is the product scalar in $\mathbb{R}^{n} ; L S=$ $\sum_{i=1}^{d} L_{i} S_{i}$, where $L_{i}$ and $S_{i}$ are the $i$ th column of $L$ and $S ; M L=\sum_{i=1}^{d} M_{i} L_{i} \in \mathbb{R}^{n} ; M(\alpha \gamma)=\sum_{i=1}^{d}\left(M_{i} \alpha\right) \gamma_{i} \in$ $\mathbb{R}^{n} ; M N=\sum_{i=1}^{d}\left(M_{i} N_{i}\right) \in \mathscr{M}_{n \times n}(\mathbb{R}) ; M Q N=$ $\sum_{i=1}^{d} M_{i} Q N_{i} \in \mathscr{M}_{n \times n}(\mathbb{R}) ; M Q \gamma=\sum_{i=1}^{d} M_{i} Q \gamma \gamma_{i} \in$ $\mathscr{M}_{n \times n}(\mathbb{R})$.

We denote by $L^{*}$ the transpose of the matrix $L$ and $M^{*}=$ $\left(M_{1}^{*}, \ldots, M_{d}^{*}\right)$.

\section{Formulation of the Problem}

Let $\left(\Omega, \mathscr{F},\left(\mathscr{F}_{t}^{(B, W)}\right)_{t \geq 0}, \mathbb{P}\right)$ be a probability space, on which a $d$-dimensional Brownian motions $W=\left(W_{t}: 0 \leq t \leq T\right)$ and $B=\left(B_{t}: 0 \leq t \leq T\right)$ are defined. For each $t \in[0, T]$, we define $\mathscr{F}_{t}^{(B, W)} \triangleq \mathscr{F}_{t, T}^{B} \vee \mathscr{F}_{t}^{W}$, where for any process $\left\{p_{t}\right\}$, $\mathscr{F}_{s, t}^{p}=\sigma\{p(r)-p(s) ; s \leq r \leq t\} \vee \mathscr{N}, \mathscr{F}_{t}^{p}=\mathscr{F}_{0, t}^{p}$. Note that the collection $\left\{\mathscr{F}_{t}, t \in[0, T]\right\}$ is neither increasing nor decreasing, and it does not constitute a classical filtration, where $\mathcal{N}$ denotes the total of $\sigma$-null sets and $\sigma_{1} \vee \sigma_{2}$ denotes the $\sigma$-fields generated by $\sigma_{1} \cup \sigma_{2}$.

Note that the collection $\left\{\left(\mathscr{F}_{t}^{(B, W)}\right)_{t}, t \in[0, T]\right\}$ is neither increasing nor decreasing, and it does not constitute a filtration.

Let $T$ be a positive real number and $U$ a nonempty subset of $\mathbb{R}^{k}$ 
2.1. The Strict Control Problem. For any $n \in \mathbb{N}$, let $\mathscr{M}^{2}(0$, $\left.T ; \mathbb{R}^{n}\right)$ denote the set of $n$-dimensional jointly measurable random processes $\left\{\varphi_{t}, t \in[0, T]\right\}$ which satisfy

$$
\text { (i) : } \mathbb{E}\left[\int_{0}^{T}\left|\varphi_{t}\right|^{2} d t\right]<\infty
$$

(ii): $\varphi_{t}$ is $\left(\mathscr{F}_{t}^{(B, W)}\right)$ measurable, for a.e. $t \in[0, T]$.

We denote similarly by $\mathcal{S}^{2}\left([0, T] ; \mathbb{R}^{n}\right)$ the set of continuous $n$-dimensional random processes which satisfy

(i) : $\mathbb{E}\left[\sup _{0 \leq t \leq T}\left|\varphi_{t}\right|^{2}\right]<\infty$,

(ii): $\varphi_{t}$ is $\left(\mathscr{F}_{t}^{(B, W)}\right)$ measurable, for any $t \in[0, T]$.

Let $T$ be a strictly positive real number and $U$ is a nonempty subset of $\mathbb{R}^{k}$.

Definition 1. $\left(y_{t}, z_{t}\right)$ is said to be a solution of (1), if and only if, $\left(y_{t}, z_{t}\right) \in \mathscr{M}^{2}\left(\mathbb{R}^{n \times d}\right) \times \mathcal{S}^{2}\left(\mathbb{R}^{n \times m}\right)$ and it satisfies (1).

Definition 2. An admissible strict control is an $\mathscr{F}_{t}$-adapted process $\nu=\left(\nu_{t}\right)$ with values in $U$ such that $\mathbb{E}\left[\sup _{t \in[0, T]}\left|\nu_{t}\right|^{2}\right]<$ $\infty$.

We denote by $\mathcal{U}$ the set of all admissible controls.

For any $\nu \in \mathcal{U}$, we consider the following BDSDE:

$$
\begin{gathered}
d y_{t}^{v}=-f\left(t, y_{t}^{v}, z_{t}^{v}, v_{t}\right) d t+g\left(t, y_{t}^{v}, z_{t}^{v}, v_{t}\right) d B_{t}+z_{t}^{v} d W_{t}, \\
y_{T}^{v}=\xi,
\end{gathered}
$$

where $f:[0, T] \times \mathbb{R}^{m} \times \mathscr{M}_{m \times d}(\mathbb{R}) \times U \rightarrow \mathbb{R}^{m}, g:[0, T] \times$ $\mathbb{R}^{m} \times \mathscr{M}_{m \times d}(\mathbb{R}) \times U \rightarrow \mathbb{R}^{m \times k}$, and $\xi$ is an $n$-dimensional $\mathscr{F}_{0}$-measurable random variable such that $\mathbb{E}|\xi|^{2}<\infty$.

The expected cost is defined from $\mathcal{U}$ into $\mathbb{R}$ by

$$
J(\nu)=\mathbb{E}\left[\Psi\left(y_{0}^{\nu}\right)+\int_{0}^{T} l\left(t, y_{t}^{\nu}, z_{t}^{v}, v_{t}\right) d t\right],
$$

where $\Psi: \mathbb{R}^{m} \rightarrow \mathbb{R}, l:[0, T] \times \mathbb{R}^{m} \times \mathscr{M}_{m \times d}(\mathbb{R}) \times U \rightarrow \mathbb{R}$.

The control problem is to minimize the functional $J$ over $\mathcal{U}$, if $u \in \mathcal{U}$ is an optimal solution; that is,

$$
J(u)=\inf _{\nu \in \mathscr{U}} J(\nu)
$$

A control that solves this problem is called optimal. Our goal is to establish a necessary condition of optimality for controls in the form of stochastic maximum principle.

The following assumptions will be in force throughout this paper:

$f, g, \Psi$, and $l$ are continuously differentiable with respect to $(y, z)$.
They and all their derivatives with respect to $(y, z)$ are continuous in $(y, z, v)$ and uniformly bounded by $C>0 ; \Psi, l$ and are bounded by $C>0$.

We assume moreover that there exist constants $C>$ 0 and $0 \leq \zeta<1$ such that for any $(w, t) \in \Omega \times$ $[0, T],\left(Y_{1}, Z_{1}\right) ;\left(Y_{2}, Z_{2}\right) \in \mathbb{R}^{m} \times \mathscr{M}_{m \times d}(\mathbb{R})$,

$$
\begin{aligned}
\text { (i) : } & \left|f\left(t, Y_{1}, Z_{1}\right)-f\left(t, Y_{2}, Z_{2}\right)\right|^{2} \\
& \leq C\left(\left|Y_{1}-Y_{2}\right|^{2}+\left\|Z_{1}-Z_{2}\right\|^{2}\right), \\
\text { (ii) : } & \left\|g\left(t, Y_{1}, Z_{1}\right)-g\left(t, Y_{2}, Z_{2}\right)\right\|^{2} \\
& \leq C\left|Y_{1}-Y_{2}\right|^{2}+\zeta\left(\left\|Z_{1}-Z_{2}\right\|^{2}\right) .
\end{aligned}
$$

Under the above assumptions, for every $v \in \mathcal{U}$, (10) has a unique strong solution and the functional cost $J$ is well defined from $\mathcal{U}$ into $\mathbb{R}$.

2.2. The Relaxed Model. The idea for the relaxed strict control problem defined above is to embed the set $U$ of strict controls into a wider class which gives a more suitable topological structure. In the relaxed model, the $U$-valued process $v$ is replaced by a $\mathbb{P}(U)$-valued process $q$, where $\mathbb{P}(U)$ denotes the space of probability measure on $U$ equipped with the topology of stable convergence.

Definition 3. A relaxed control $\left(q_{t}\right)_{t}$ is a $\mathbb{P}(U)$-valued process, progressively measurable with respect to $\left(\mathscr{F}_{t}\right)_{t}$ and such that for each $t, \mathbf{1}_{0, t}\left[q_{t}\right.$ is $\mathscr{F}_{t}$-measurable.

We denote by $\mathscr{R}$ the set of all admissible relaxed controls.

Remark 4. Every relaxed control $q$ may be disintegrated as $q(d t, d a)=q(t, d a) d t=q_{t}(d a) d t$, where $q_{t}(d a)$ is a progressively measurable process with value in the set of probability measures $\mathbb{P}(U)$. The set $U$ is embedded into the set $\mathscr{R}$ of relaxed process by the mapping $f: v \in \mathcal{U} \mapsto$ $f_{v}(d t, d a)=\delta_{v_{t}}(d a) d t \in \mathscr{R}$, where $\delta_{v}$ is the atomic measure concentrated at a single point $v$.

For any $q \in \mathscr{R}$, we consider the following relaxed BDSDE:

$$
\begin{gathered}
d y_{t}^{q}=-\int_{U} f\left(t, y_{t}^{q}, z_{t}^{q}, a\right) q_{t}(d a) d t \\
+\int_{U} g\left(t, y_{t}^{q}, z_{t}^{q}, a\right) q_{t}(d a) d B_{t}+z_{t}^{q} d W_{t}, \\
y_{T}^{q}=\xi .
\end{gathered}
$$

The expected cost to be minimized, the relaxed model, is defined from $\mathscr{R}$ into $\mathbb{R}$ by

$$
\mathscr{J}(q)=\mathbb{E}\left[\Psi\left(y_{0}^{q}\right)+\int_{0}^{T} \int_{U} l\left(t, y_{t}^{q}, z_{t}^{q}, a\right) q_{t}(d a) d t\right] .
$$

A relaxed control $\rho$ is called optimal if it solves

$$
\mathscr{J}(\mu)=\inf _{q \in \mathscr{R}} \mathscr{J}(q) .
$$

Existence of an optimal solution for the problem $\{(14)$, (15), (16)\} has been solved. 


\section{Optimality Conditions for Relaxed Controls}

In this section, we study the problem $\{(14),(15),(16)\}$ and we establish necessary condition of optimality for relaxed controls.

3.1. Preliminary Results. Since the set of relaxed controls $\mathscr{R}$ is convex, then the classical way of treating such a problem is to use the convex perturbation method. More precisely, let $\mu$ be an optimal relaxed control and $\left(y_{t}^{\mu}, z_{t}^{\mu}\right)$ the solution of (14) controlled by $\mu$. Then, we can define a perturbation relaxed as follows:

$$
\mu_{t}^{\theta}=\mu_{t}+\theta\left(q_{t}-\mu_{t}\right)
$$

where $\theta>0$ is sufficiently small and $q$ is an arbitrary element of $\mathscr{R}$.

Denote by $\left(y_{t}^{\theta}, z_{t}^{\theta}\right)$ the solution of (14) associated with $\mu^{\theta}$.

From the optimality of $\mu$, the variational inequality will be derived from the fact that

$$
0 \leq \mathscr{J}\left(\mu^{\theta}\right)-\mathscr{J}(\mu)
$$

To this end, we need the following classical Lemmas.

Lemma 5. Under the assumption (13) and (Hypo02), we have

$$
\begin{aligned}
& \lim _{\theta \rightarrow 0}\left[\sup _{0 \leq t \leq T} \mathbb{E}\left|y_{t}^{\theta}-y_{t}^{\mu}\right|^{2}\right]=0, \\
& \lim _{\theta \rightarrow 0} \mathbb{E}\left[\int_{0}^{T}\left|z_{t}^{\theta}-z_{t}^{\mu}\right|^{2} d t\right]=0 .
\end{aligned}
$$

Proof. Let us prove (19) and (20).

Applying Itô's formula to $\left(y_{t}^{\theta}-y_{t}^{\mu}\right)^{2}$, and since $\iint\left(y_{t}^{\theta}-\right.$ $\left.y_{t}^{\mu}\right)\left(z_{s}^{\theta}-z_{s}^{\mu}\right) d W_{s}$ is martingale; then taking the expectation for any $s \leq T$, we have

$$
\begin{gathered}
\mathbb{E}\left|y_{t}^{\theta}-y_{t}^{\mu}\right|^{2}+\mathbb{E} \int_{t}^{T}\left|z_{s}^{\theta}-z_{s}^{\mu}\right|^{2} d s \\
=2 \mathbb{E} \int_{t}^{T} \mid\left(y_{s}^{\theta}-y_{s}^{\mu}\right)\left[\int_{U} f\left(s, y_{s}^{\theta}, z_{s}^{\theta}, a\right) \mu_{s}^{\theta}(d a)\right. \\
\left.\quad-\int_{U} f\left(s, y_{s}^{\mu}, z_{s}^{\mu}, a\right) \mu_{s}(d a)\right] \mid d s \\
+\mathbb{E} \int_{t}^{T} \mid\left[\int_{U} g\left(s, y_{s}^{\theta}, z_{s}^{\theta}, a\right) \mu_{s}^{\theta}(d a)\right. \\
\left.-\int_{U} g\left(s, y_{s}^{\mu}, z_{s}^{\mu}, a\right) \mu_{s}(d a)\right] \mid d s .
\end{gathered}
$$

From the Young formula, for every $\varepsilon>0, a b \leq(1 /(2) 1+$ $\alpha)) a^{2}+((1+\alpha) / 2) b^{2}$, and by using the definition of $\mu_{s}^{\theta}$, we have

$$
\begin{aligned}
& \mathbb{E}\left|y_{t}^{\theta}-y_{t}^{\mu}\right|^{2}+\mathbb{E} \int_{t}^{T}\left|z_{s}^{\theta}-z_{s}^{\mu}\right|^{2} d s \\
& \leq(1+\alpha) \mathbb{E} \int_{t}^{T}\left|\left(y_{s}^{\theta}-y_{s}^{\mu}\right)\right|^{2} d s \\
& +\theta^{2} \frac{C}{(1+\alpha)} \mathbb{E} \int_{t}^{T} \mid \int_{U} f\left(s, y_{s}^{\theta}, z_{s}^{\theta}, a\right) q_{s}(d a) \\
& -\left.\int_{U} f\left(s, y_{s}^{\theta}, z_{s}^{\theta}, a\right) \mu_{s}(d a)\right|^{2} d s \\
& +\theta^{2} C \mathbb{E} \int_{t}^{T} \mid \int_{U} g\left(s, y_{s}^{\theta}, z_{s}^{\theta}, a\right) q_{s}(d a) \\
& -\left.\int_{U} g\left(s, y_{s}^{\theta}, z_{s}^{\theta}, a\right) \mu_{s}(d a)\right|^{2} d s \\
& +\frac{C}{(1+\alpha)} \mathbb{E} \int_{t}^{T} \mid \int_{U} f\left(s, y_{s}^{\theta}, z_{s}^{\theta}, a\right) \mu_{s}(d a) \\
& -\left.\int_{U} f\left(s, y_{s}^{\mu}, z_{s}^{\theta}, a\right) \mu_{s}(d a)\right|^{2} d s \\
& +\frac{C}{(1+\alpha)} \mathbb{E} \int_{t}^{T} \mid \int_{U} f\left(s, y_{s}^{\mu}, z_{s}^{\theta}, a\right) \mu_{s}(d a) \\
& -\left.\int_{U} f\left(s, y_{s}^{\mu}, z_{s}^{\mu}, a\right) \mu_{s}(d a)\right|^{2} d s \\
& +C \mathbb{E} \int_{t}^{T} \mid \int_{U} g\left(s, y_{s}^{\theta}, z_{s}^{\theta}, a\right) \mu_{s}(d a) \\
& -\left.\int_{U} g\left(s, y_{s}^{\mu}, z_{s}^{\theta}, a\right) \mu_{s}(d a)\right|^{2} d s \\
& +C \mathbb{E} \int_{t}^{T} \mid \int_{U} g\left(s, y_{s}^{\mu}, z_{s}^{\mu}, a\right) \mu_{s}(d a) \\
& -\left.\int_{U} g\left(s, y_{s}^{\mu}, z_{s}^{\mu}, a\right) \mu_{s}(d a)\right|^{2} d s .
\end{aligned}
$$

Since $g$ and $f$ are uniformly Lipschitz with respect to $y$ and $z$ and from (Hypo02), where $0 \leq \zeta<1$, then

$$
\begin{aligned}
\mathbb{E} \mid y_{t}^{\theta} & -\left.y_{t}^{\mu}\right|^{2}+\left(1-\frac{C}{(1+\alpha)}-\zeta\right) \mathbb{E} \int_{t}^{T}\left|z_{s}^{\theta}-z_{s}^{\mu}\right|^{2} d s \\
& \leq\left(\frac{C}{(1+\alpha)}+(1+\alpha)+C\right) \mathbb{E} \int_{t}^{T}\left|\left(y_{s}^{\theta}-y_{s}^{\mu}\right)\right|^{2} d s+M^{\theta}
\end{aligned}
$$

where $M^{\theta}$ is given by $M^{\theta}=C\left((1+\alpha)^{-1}+1\right) \theta^{2}$, we have

$$
M^{\theta}=o(\theta)
$$


Let

$$
\alpha= \begin{cases}0, & C \leq \frac{1-\zeta}{2}, \\ \frac{2 c+\zeta-1}{1-\zeta}, & C>\frac{1-\zeta}{2} .\end{cases}
$$

Then, $1-C /(1+\alpha)-\zeta \geq(1-\zeta) / 2>0$.

Choose $c_{1}=1-C /(1+\alpha)-\zeta, c_{2}=C /(1+\alpha)+(1+\alpha)+C$, then we have

$$
\begin{aligned}
\mathbb{E} \mid y_{t}^{\theta} & -\left.y_{t}^{\mu}\right|^{2}+c_{1} \mathbb{E} \int_{t}^{T}\left|z_{s}^{\theta}-z_{s}^{\mu}\right|^{2} d s \\
& \leq c_{2} \mathbb{E} \int_{t}^{T}\left|\left(y_{s}^{\theta}-y_{s}^{\mu}\right)\right|^{2} d s+M^{\theta} .
\end{aligned}
$$

From the above inequality, we derive the following two inequalities:

$$
\begin{gathered}
\mathbb{E}\left|y_{t}^{\theta}-y_{t}^{\mu}\right| \leq c_{2} \mathbb{E} \int_{t}^{T}\left|\left(y_{s}^{\theta}-y_{s}^{\mu}\right)\right|^{2} d s+M^{\theta} \\
\mathbb{E} \int_{t}^{T}\left|z_{s}^{\theta}-z_{s}^{\mu}\right|^{2} d s \leq \frac{c_{2}}{c_{1}} \mathbb{E} \int_{t}^{T}\left|\left(y_{s}^{\theta}-y_{s}^{\mu}\right)\right|^{2} d s+\frac{1}{c_{1}} M^{\theta} .
\end{gathered}
$$

By using (24), Gronwall's lemma, and Burkholder-DavisGundy inequality in (27), we obtain (19). Finally, (20) is derived from (27), (28), and (24).

Lemma 6. Let $\tilde{y}_{t}$ be the solution of the following linear equation (called variational equation):

$$
\begin{aligned}
d \tilde{y}_{t}= & -\int_{U}\left[f_{y}\left(t, y_{t}^{\mu}, z_{t}^{\mu}, a\right) \cdot \tilde{y}_{t}\right] \mu(d a) d t \\
& -\int_{U}\left[f_{z}\left(t, y_{t}^{\mu}, z_{t}^{\mu}, a\right) \cdot \widetilde{z}_{t}\right] \mu(d a) d t \\
& +\left[\int_{U} f\left(t, y_{t}^{\mu}, z_{t}^{\mu}, a\right) \mu_{s}(d a)\right. \\
& \left.+\int_{U} f\left(t, y_{t}^{\mu}, z_{t}^{\mu}, a\right) q_{t}(d a)\right] d t \\
& +\int_{U}\left[g_{z}\left(t, y_{t}^{\mu}, y_{t}^{\mu}, z_{t}^{\mu}, a\right) \cdot \tilde{y}_{t}\right] \mu(d a) d t \\
& +\left[\int_{U} g\left(t, y_{t}^{\mu}, z_{t}^{\mu}, a\right) q_{s}(d a)\right. \\
& \left.-\int_{U} g\left(t, y_{t}^{\mu}, z_{t}^{\mu}, a\right) \mu_{t}(d a)\right] d t \\
+ & \widetilde{z}_{t} d W_{t}, \\
& \tilde{y}_{0}=0 .
\end{aligned}
$$

Then, we have

$$
\begin{aligned}
& \lim _{\theta \rightarrow 0}\left[\sup _{0 \leq t \leq T} \mathbb{E}\left|\frac{y_{t}^{\theta}-y_{t}^{\mu}}{\theta}-\tilde{y}_{t}\right|^{2}\right]=0, \\
& \lim _{\theta \rightarrow 0} \mathbb{E}\left[\int_{0}^{T}\left|\frac{z_{t}^{\theta}-z_{t}^{\mu}}{\theta}-\widetilde{z}_{t}\right|^{2} d t\right]=0 .
\end{aligned}
$$

Proof. For simplicity, we put $Y_{t}^{\theta}=\left(y_{t}^{\theta}-y_{t}^{\mu}\right) / \theta-\tilde{y}_{t}, Z_{t}^{\theta}=$ $\left(z_{t}^{\theta}-z_{t}^{\mu}\right) / \theta-\widetilde{z}_{t}$.

(i) Proof of (30): applying Itô's formula to $\left(y_{t}^{\theta}-y_{t}^{\mu}\right)^{2}$, and from the Young formula, for every $\varepsilon>0$, we have

$$
\begin{aligned}
& \mathbb{E}\left|Y_{t}^{\theta}\right|^{2}+\mathbb{E} \int_{t}^{T}\left|Z_{s}^{\theta}\right|^{2} d s \\
& \leq \frac{1}{\varepsilon} \mathbb{E} \int_{t}^{T}\left|Y_{s}^{\theta}\right|^{2} d s \\
& +\varepsilon \mathbb{E} \int_{t}^{T} \mid\left[\int_{U} f\left(s, y_{s}^{\theta}, z_{s}^{\theta}, a\right) \mu_{s}^{\theta}(d a)\right. \\
& -\int_{U} f\left(s, y_{s}^{\mu}, z_{s}^{\mu}, a\right) \mu_{s}(d a) \\
& +\int_{U}\left[f_{y}\left(s, y_{s}^{\mu}, z_{s}^{\mu}, a\right) \cdot \tilde{y}_{s}\right. \\
& \left.+f_{z}\left(s, y_{s}^{\mu}, z_{s}^{\mu}, a\right) \cdot \widetilde{z}_{s}\right] \mu(d a) \\
& -\left(\int_{U} f\left(s, y_{s}^{\mu}, z_{s}^{\mu}, a\right) \mu_{s}(d a)\right. \\
& \left.\left.-\int_{U} f\left(s, y_{s}^{\mu}, z_{s}^{\mu}, a\right) q_{s}(d a)\right)\right]\left.\right|^{2} d s \\
& +\varepsilon \mathbb{E} \int_{t}^{T} \mid\left[\int_{U} g\left(s, y_{s}^{\theta}, z_{s}^{\theta}, a\right) \mu_{s}^{\theta}(d a)\right. \\
& -\int_{U} g\left(s, y_{s}^{\mu}, z_{s}^{\mu}, a\right) \mu_{s}(d a) \\
& +\int_{U}\left[g_{y}\left(s, y_{s}^{\mu}, z_{s}^{\mu}, a\right) \cdot \tilde{y}_{s}\right. \\
& \left.+g_{z}\left(s, y_{s}^{\mu}, z_{s}^{\mu}, a\right) \cdot \widetilde{z}_{s}\right] \mu(d a) \\
& -\left(\int_{U} g\left(s, y_{s}^{\mu}, z_{s}^{\mu}, a\right) \mu_{s}(d a)\right. \\
& \left.\left.-\int_{U} g\left(s, y_{s}^{\mu}, z_{s}^{\mu}, a\right) q_{s}(d a)\right)\right]\left.\right|^{2} d s .
\end{aligned}
$$


For simplicity, we put $\Lambda_{t}^{\theta}(a)=\left(t, y_{t}^{\mu}+\alpha \theta\left(Y_{t}^{\theta}+\tilde{y}_{t}\right), z_{t}^{\mu}+\right.$ $\left.\alpha \theta\left(Z_{t}^{\theta}+\widetilde{z}_{t}\right), a\right)$, we have the following inequality:

$$
\begin{aligned}
\mathbb{E}\left|Y_{t}^{\theta}\right|^{2} & +\mathbb{E} \int_{t}^{T}\left|Z_{s}^{\theta}\right|^{2} d s \\
\leq & \frac{1}{\varepsilon} \mathbb{E} \int_{t}^{T}\left|Y_{s}^{\theta}\right|^{2} d s+\varepsilon \mathbb{E} \int_{t}^{T}\left|F_{s}^{y} Y_{s}^{\theta}+F_{s}^{z} Z_{s}^{\theta}-\eta_{s}^{\theta}\right|^{2} d s \\
& +\varepsilon \mathbb{E} \int_{t}^{T}\left|G_{s}^{y} Y_{s}^{\theta}+G_{s}^{z} Z_{s}^{\theta}-\eta_{s}^{\theta}\right|^{2} d s
\end{aligned}
$$

where

$$
\begin{gathered}
F_{t}^{y}=-\int_{0}^{1} \int_{U} f_{y}\left(\Lambda_{t}^{\theta}(a)\right) \mu_{t}(d a) d \alpha, \\
G_{t}^{y}=\int_{0}^{1} \int_{U} g_{y}\left(\Lambda_{t}^{\theta}(a)\right) \mu_{t}(d a) d \alpha, \\
F_{t}^{z}=-\int_{0}^{1} \int_{U} f_{z}\left(\Lambda_{t}^{\theta}(a)\right) \mu_{t}(d a) d \alpha, \\
G_{t}^{z}=\int_{0}^{1} \int_{U} g_{z}\left(\Lambda_{t}^{\theta}(a)\right) \mu_{t}(d a) d \alpha,
\end{gathered}
$$

and $\eta_{t}^{\theta}, \eta_{t}^{\prime \theta}$ are given by

$$
\begin{aligned}
& \eta_{t}^{\theta}=\int_{t}^{T} \int_{U}\left[f_{y}\left(\Lambda_{s}^{\theta}(a)\right)\left(y_{s}^{\theta}-y_{s}^{\mu}\right)\right. \\
&+\left.f_{z}\left(\Lambda_{s}^{\theta}(a)\right)\left(z_{s}^{\theta}-z_{s}^{\mu}\right)\right] q_{s}(d a) d s \\
&-\int_{t}^{T} \int_{U}[ f_{y}\left(\Lambda_{s}^{\theta}(a)\right)\left(y_{s}^{\theta}-y_{s}^{\mu}\right) \\
&\left.+f_{z}\left(\Lambda_{s}^{\theta}(a)\right)\left(z_{s}^{\theta}-z_{s}^{\mu}\right)\right] \mu_{s}(d a) d s, \\
& \eta_{t}^{\prime \theta}=\int_{t}^{T} \int_{U}\left[g_{y}\left(\Lambda_{s}^{\theta}(a)\right)\left(y_{s}^{\theta}-y_{s}^{\mu}\right)\right. \\
&\left.+g_{z}\left(\Lambda_{s}^{\theta}(a)\right)\left(z_{s}^{\theta}-z_{s}^{\mu}\right)\right] q_{s}(d a) d s \\
&-\int_{t}^{T} \int_{U}\left[g_{y}\left(\Lambda_{s}^{\theta}(a)\right)\left(y_{s}^{\theta}-y_{s}^{\mu}\right)\right. \\
&\left.+g_{z}\left(\Lambda_{s}^{\theta}(a)\right)\left(z_{s}^{\theta}-z_{s}^{\mu}\right)\right] \mu_{s}(d a) d s .
\end{aligned}
$$

Since $F_{t}^{y}, F_{t}^{z}, G_{t}^{y}$, and $G_{t}^{z}$ are bounded, then

$$
\begin{aligned}
\mathbb{E}\left|Y_{t}^{\theta}\right|^{2} & +\mathbb{E} \int_{t}^{T}\left|Z_{s}^{\theta}\right|^{2} d s \\
\leq & \frac{1}{\varepsilon} \mathbb{E} \int_{t}^{T}\left|Y_{s}^{\theta}\right|^{2} d s+\varepsilon C \mathbb{E} \int_{t}^{T}\left|Y_{s}^{\theta}\right|^{2} d s \\
& +\varepsilon C \mathbb{E} \int_{\Gamma}\left|Z_{s}^{\theta}\right|^{2} d s+\Pi_{t}^{\theta},
\end{aligned}
$$

where $\Pi_{t}^{\theta}=\varepsilon C \mathbb{E} \int_{t}^{T}\left|\eta_{s}^{\theta}\right|^{2} d s+\varepsilon C \mathbb{E} \int_{t}^{T}\left|\eta_{s}^{\prime \theta}\right|^{2} d s$. Choose $\varepsilon=$ $1 / 2 C$, then we have

$$
\mathbb{E}\left|Y_{t}^{\theta}\right|^{2}+\frac{1}{2} \mathbb{E} \int_{t}^{T}\left|Z_{s}^{\theta}\right|^{2} d s \leq\left(2 C+\frac{1}{2}\right) \mathbb{E} \int_{t}^{T}\left|Y_{s}^{\theta}\right|^{2} d s+\Pi_{t}^{\theta}
$$

From the above inequality, we deduce the following two inequalities:

$$
\begin{gathered}
\mathbb{E}\left|Y_{t}^{\theta}\right|^{2} \leq\left(2 C+\frac{1}{2}\right) \mathbb{E} \int_{t}^{T}\left|Y_{s}^{\theta}\right|^{2} d s+\Pi_{t}^{\theta}, \\
\mathbb{E} \int_{t}^{T}\left|Z_{s}^{\theta}\right|^{2} d s \leq 2\left(2 C+\frac{1}{2}\right) \mathbb{E} \int_{t}^{T}\left|Y_{s}^{\theta}\right|^{2} d s+2 \Pi_{t}^{\theta} .
\end{gathered}
$$

Since $f_{y}$ and $f_{z}$ and $g_{y}$ and $g_{z}$ are continuous and bounded, then from (19) and (20), we have

$$
\lim _{\theta \rightarrow 0}\left(\mathbb{E} \int_{t}^{T}\left|\eta_{s}^{\theta}\right|^{2} d s+\mathbb{E} \int_{t}^{T}\left|\eta_{s}^{\prime \theta}\right|^{2} d s\right)=0
$$

From (40), we deduce that

$$
\lim _{\theta \rightarrow 0} \Pi_{t}^{\theta}=0 .
$$

By using (33), (41), Gronwall's Lemma, and BurkholderDavis-Gundy inequality, we obtain (30). Finally, (31) is derived from (39), (41), and (30).

Lemma 7. Let $\mu$ be an optimal relaxed control minimizing the cost $\mathscr{J}$ over $\mathscr{R}$ and $\left(y_{t}^{\mu}, z_{t}^{\mu}\right)$ the associated optimal trajectory. Then, for any $q \in \mathscr{R}$, we have

$$
\begin{aligned}
& 0 \leq \mathbb{E}\left[\Psi_{y}\left(y_{0}^{\mu}\right) \cdot \tilde{y}_{0}\right] \\
&+\mathbb{E} \int_{0}^{T}\left[\int_{U} l\left(t, y_{t}^{\mu}, z_{t}^{\mu}, a\right) q_{t}(d a)\right. \\
&\left.\quad-\int_{U} l\left(t, y_{t}^{\mu}, z_{t}^{\mu}, a\right) \mu_{s}(d a)\right] d t \\
&+\mathbb{E} \int_{0}^{T}\left[\int_{U} l_{y}\left(t, y_{t}^{\mu}, z_{t}^{\mu}, a\right) \mu_{s}(d a) \cdot \tilde{y}_{t}\right] d t \\
&+\mathbb{E} \int_{0}^{T}\left[\int_{U} l_{z}\left(t, y_{t}^{\mu}, z_{t}^{\mu}, a\right) \mu_{t}(d a) \cdot \widetilde{z}_{t}\right] d t .
\end{aligned}
$$

Proof. Let $\mu$ be an optimal relaxed control minimizing the cost $\mathscr{J}$ over $\mathscr{R}$, then from (18), and by using the definition of $\mu_{t}^{\theta}$, we have

$$
\begin{aligned}
& 0 \leq \mathbb{E}\left[\Psi\left(y_{0}^{\theta}\right)-\Psi\left(y_{0}^{\mu}\right)\right] \\
&+\theta \mathbb{E} \int_{0}^{T}\left[\int_{U} l\left(t, y_{t}^{\theta}, z_{t}^{\theta}, a\right) q_{t}(d a)\right. \\
&\left.\quad-\int_{U} l\left(t, y_{t}^{\theta}, z_{t}^{\theta}, a\right) \mu_{t}(d a)\right] d t \\
&+\mathbb{E} \int_{0}^{T} \int_{U}\left[l\left(t, y_{t}^{\theta}, z_{t}^{\theta}, a\right)\right. \\
&\left.\quad-l\left(t, y_{t}^{\mu}, z_{t}^{\mu}, a\right)\right] \mu_{t}(d a) d t .
\end{aligned}
$$


Then,

$$
\begin{gathered}
0 \leq \mathbb{E} \int_{0}^{1}\left[\Psi_{y}\left(y_{0}^{\mu}+\alpha \theta\left(\tilde{y}_{0}+Y_{0}^{\theta}\right)\right) \tilde{y}_{0}\right] d \alpha \\
+\mathbb{E} \int_{0}^{T} \int_{0}^{1} \int_{U}\left[l_{y}\left(\bar{\Lambda}_{t}^{\theta}(a)\right) \cdot \tilde{y}_{t}\right. \\
\left.+l_{z}\left(\bar{\Lambda}_{t}^{\theta}(a)\right) \cdot \tilde{z}_{t}\right] \mu_{t}(d a) d \alpha d t \\
+\mathbb{E} \int_{0}^{T}\left[\int_{U} l\left(t, y_{t}^{\mu}, z_{t}^{\mu}, a\right) q_{t}(d a)\right. \\
\left.-\int_{U} l\left(t, y_{t}^{\mu}, z_{t}^{\mu}, a\right) \mu_{t}(d a)\right] d t+\beta_{t}^{\theta},
\end{gathered}
$$

where $\beta_{t}^{\theta}$ is given by

$$
\begin{gathered}
\beta_{t}^{\theta}=\mathbb{E} \int_{0}^{1}\left[\Psi_{y}\left(y_{0}^{\mu}+\alpha \theta\left(\tilde{y}_{0}+Y_{0}^{\theta}\right)\right) \cdot Y_{0}^{\theta}\right] d \alpha \\
+\mathbb{E} \int_{0}^{T} \int_{0}^{1} \int_{U}\left[l_{y}\left(\bar{\Lambda}_{t}^{\theta}(a)\right) \cdot Y_{t}^{\theta}\right. \\
\left.+l_{z}\left(\bar{\Lambda}_{t}^{\theta}(a)\right) \cdot Z_{t}^{\theta}\right] \mu_{t}(d a) d t \\
+\mathbb{E} \int_{0}^{T} \int_{0}^{1} \int_{U}\left[l_{y}\left(\bar{\Lambda}_{t}^{\theta}(a)\right)\left(y_{t}^{\theta}-y_{t}^{\mu}\right)\right. \\
\left.+l_{z}\left(\bar{\Lambda}_{t}^{\theta}(a)\right)\left(z_{t}^{\theta}-z_{t}^{\mu}\right)\right] q_{t}(d a) d t \\
-\mathbb{E} \int_{0}^{T} \int_{0}^{1} \int_{U}\left[l_{y}\left(\bar{\Lambda}_{t}^{\theta}(a)\right)\left(y_{t}^{\theta}-y_{t}^{\mu}\right)\right. \\
\left.\quad+l_{z}\left(\bar{\Lambda}_{t}^{\theta}(a)\right)\left(z_{t}^{\theta}-z_{t}^{\mu}\right)\right] \mu_{t}(d a) d t .
\end{gathered}
$$

For simplicity, we put $\bar{\Lambda}_{t}^{\theta}(a)=\left(t, y_{t}^{\mu}+\alpha \theta\left(Y_{t}^{\theta}+\tilde{y}_{t}\right), z_{t}^{\mu}+\right.$ $\left.\alpha \theta\left(Z_{t}^{\theta}+\widetilde{z}_{t}\right), a\right)$. Since the derivatives $\Psi_{y}, l_{y}, l_{z}$ are continuous and bounded, then by using (30), (31), (19), and (20) and the Cauchy-Schwartz inequality, we have $\lim _{\theta \rightarrow 0} \beta_{t}^{\theta}=0$. By letting $\theta$ go to 0 in (44), the proof is completed.

3.2. Necessary Optimality Conditions for Relaxed Controls. Starting from the variational inequality (42), we can now state necessary optimality conditions for the relaxed control problem $\{(14),(15),(16)\}$ in the global form.

The Hamiltonian $\mathscr{H}$ is defined from $[0, T] \times \mathbb{R}^{m} \times$ $\mathscr{M}_{n \times m}(\mathbb{R}) \times \mathbb{P}(U) \times \mathscr{M}_{n \times d}(\mathbb{R})$ into $\mathbb{R}$ by

$$
\begin{aligned}
& \mathscr{H}(t, y, z, \mu, Q, R) \\
& =\int_{U} l(t, y, z, a) \mu_{t}(d a)+Q \cdot \int_{U} f(t, y, z, a) \mu_{t}(d a) \\
& \quad+R \cdot \int_{U} g\left(t, y_{t}, z_{t}, a\right) \mu_{t}(d a) .
\end{aligned}
$$

Theorem 8 (necessary optimality conditions for relaxed controls). Let $\mu$ be an optimal relaxed control minimizing the functional $\mathcal{J}$ over $\mathscr{R}$ and $\left(y_{t}^{\mu}, z_{t}^{\mu}\right)$ the solution of (14) associated with $\mu$. Then, there exists unique adapted process $\left(Q^{\mu}\right) \in L_{\mathscr{F}}^{2}\left([0, T] ; \mathbb{R}^{m}\right)$ of the following FDSDE system (called adjoint equation):

$$
\begin{aligned}
& d Q_{t}=-\left[\int_{U} l_{y}\left(t, y_{t}^{\mu}, z_{t}^{\mu}, a\right) \mu_{t}(d a)\right. \\
&+\int_{U} f_{y}\left(t, y_{t}^{\mu}, z_{t}^{\mu}, a\right) \mu_{t}(d a) Q_{t}^{\mu} \\
&\left.-\int_{U} g_{y}\left(t, y_{t}^{\mu}, z_{t}^{\mu}, a\right) \mu_{t}(d a) R_{t}^{\mu}\right] d t \\
&- {\left[\int_{U} l_{z}\left(t, y_{t}^{\mu}, z_{t}^{\mu}, a\right) \mu_{t}(d a)\right.} \\
&+\int_{U} f_{z}\left(t, y_{t}^{\mu}, z_{t}^{\mu}, a\right) \mu_{t}(d a) Q_{t}^{\mu} \\
&\left.-\int_{U} g_{z}\left(t, y_{t}^{\mu}, z_{t}^{\mu}, a\right) \mu_{t}(d a) R_{t}^{\mu}\right] d W_{t} \\
&- R_{t}^{\mu} d B_{t}, \\
& Q_{0}^{\mu}=\Psi_{y}\left(y^{\mu}(0)\right)
\end{aligned}
$$

such that for every $q_{t} \in \mathbb{P}(U)$,

$$
\mathscr{H}\left(t, y_{t}^{\mu}, z_{t}^{\mu}, q_{t}, Q_{t}^{\mu}, R_{t}^{\mu}\right) \geq \mathscr{H}\left(t, y_{t}^{\mu}, z_{t}^{\mu}, \mu_{t}, Q_{t}^{\mu}, R_{t}^{\mu}\right)
$$

$\forall \mu_{t} \in \mathbb{P}(U)$, ae, as.

Proof. Since $Q_{0}^{\mu}=\Psi_{y}\left(y_{0}^{\mu}\right)$, then (42) becomes

$$
\begin{aligned}
0 \leq & \mathbb{E}\left[Q_{0}^{\mu} \widetilde{y}_{0}\right] \\
+ & \mathbb{E} \int_{0}^{T}\left[\int_{U} l^{\mu}\left(t, y_{t}^{\mu}, z_{t}^{\mu}, a\right) q_{t}(d a)\right. \\
& \left.\quad-\int_{U} l^{\mu}\left(t, y_{t}^{\mu}, z_{t}^{\mu}, a\right) \mu_{s}(d a)\right] d t \\
+ & \int_{0}^{T}\left[\int_{U} l_{y}^{\mu}\left(t, y_{t}^{\mu}, z_{t}^{\mu}, a\right) \mu_{s}(d a) \cdot \tilde{y}_{t}\right] d t \\
+ & \mathbb{E} \int_{0}^{T}\left[\int_{U} l_{z}^{\mu}\left(t, y_{t}^{\mu}, z_{t}^{\mu}, a\right) \mu_{t}(d a) \cdot \widetilde{z}_{t}\right] d t .
\end{aligned}
$$


By applying Itô's formula to $\left(Q_{t}^{\mu} \tilde{y}_{t}\right)$, we get

$$
\begin{aligned}
\mathbb{E}\left[Q_{0}^{\mu} \tilde{y}_{0}\right]= & \mathbb{E}\left[Q_{T}^{\mu} \tilde{y}_{T}\right] \\
& -\mathbb{E}\left[\int_{0}^{T} \int_{U} l_{y}^{\mu}(t, a) \mu_{t}(d a) \cdot \tilde{y}_{t}\right] d t \\
& -\mathbb{E}\left[\int_{0}^{T} \int_{U} l_{z}^{\mu}(t, a) \mu_{t}(d a) \cdot \widetilde{z}_{t}\right] d t \\
& +\mathbb{E} \int_{0}^{T} Q_{t}^{\mu}\left[\int_{U} f^{\mu}(t, a) q_{t}(d a)\right. \\
& +\mathbb{E} \int_{0}^{T} R_{t}^{\mu}\left[\int_{U} g^{\mu}(t, a) q_{t}(d a)\right. \\
\left.-\int_{U}^{\mu}(t, a) \mu_{t}(d a)\right] d t & \left.g^{\mu}(t, a) \mu_{t}(d a)\right] d t .
\end{aligned}
$$

Then, for every $q \in \mathscr{R}$, (49) becomes

$$
\begin{aligned}
0 \leq \mathbb{E} \int_{0}^{T}[ & \mathscr{H}\left(t, y_{t}^{\mu}, z_{t}^{\mu}, q_{t}, Q_{t}^{\mu}, R_{t}^{\mu}\right) \\
& \left.\quad \mathscr{H}\left(t, y_{t}^{\mu}, z_{t}^{\mu}, \mu_{t}, Q_{t}^{\mu}, R_{t}^{\mu}\right)\right] d t .
\end{aligned}
$$

Now, let $\mu \in \mathscr{R}$ and $F$ be an arbitrary element of the $\sigma$ algebra $\mathscr{F}_{t}$, and set $\phi=q_{t} \mathbf{1}_{F}+\mu_{t} \mathbf{1}_{\Omega-F}$. It is obvious that $\phi$ is an admissible relaxed control.

Applying the above inequality with $\phi$, we get

$$
\begin{array}{r}
0 \leq \mathbb{E}\left[\mathbf { 1 } _ { F } \left\{\mathscr{H}\left(t, y_{t}^{\mu}, z_{t}^{\mu}, q_{t}, Q_{t}^{\mu}, R_{t}^{\mu}\right)\right.\right. \\
\left.\left.-\mathscr{H}\left(t, y_{t}^{\mu}, z_{t}^{\mu}, \mu_{t}, Q_{t}^{\mu}, R_{t}^{\mu}\right)\right\}\right], \\
\forall F \in \mathscr{F}_{t} .
\end{array}
$$

Which implies that

$0 \leq \mathbb{E}\left[\frac{\mathscr{H}\left(t, y_{t}^{\mu}, z_{t}^{\mu}, q_{t}, Q_{t}^{\mu}, R_{t}^{\mu}\right)-\mathscr{H}\left(t, y_{t}^{\mu}, z_{t}^{\mu}, \mu_{t}, Q_{t}^{\mu}, R_{t}^{\mu}\right)}{\mathscr{F}_{t}}\right]$.

The quantity inside the conditional expectation is $\mathscr{F}_{t^{-}}$ measurable, and thus the result follows immediately.

\section{Sufficient Optimality Conditions for Relaxed Controls}

In this section, we study when necessary optimality conditions (48) become sufficient. For any $q \in \mathscr{R}$, we denote by $\left(y^{q}, z^{q}\right)$ the solution of (14) controlled by $q$.

Theorem 9 (sufficient optimality conditions for relaxed controls). Assume that the functions $\Psi$ and $(y, z) \mapsto$ $\mathscr{H}(t, y, z, q, Q, R)$ are convex, and for any $q \in \mathscr{R}, y_{T}^{q}=0$ is an $m$-dimensional $\mathscr{F}_{t}$-measurable random variable such that $\mathbb{E}|\xi|^{2}<\infty$. Then, $\mu$ is an optimal solution of the relaxed control problem \{(14), (15), (16)\}, if it satisfies (48).
Proof. Let $\mu_{2}$ be an arbitrary element of $\mathscr{R}$ (candidate to be optimal). For any $\mu_{1} \in \mathscr{R}$, we have

$$
\begin{aligned}
\mathscr{J}\left(\mu_{1}\right)-\mathscr{J}\left(\mu_{2}\right) & \\
= & \mathbb{E}\left[h\left(y_{0}^{\mu_{1}}\right)-h\left(y_{0}^{\mu_{2}}\right)\right] \\
+ & \int_{0}^{T}\left[\int_{U} l\left(t, y_{t}^{\mu_{1}}, z_{t}^{\mu_{1}}, a\right) \mu_{1 t}(d a)\right. \\
& \left.-\int_{U} l\left(t, y_{t}^{\mu_{2}}, z_{t}^{\mu_{2}}, a\right) \mu_{2 t}(d a)\right] d t .
\end{aligned}
$$

Since $\Psi$ are convex, then

$$
\Psi\left(y_{0}^{\mu_{1}}\right)-\Psi\left(y_{0}^{\mu_{2}}\right) \geq \Psi_{y}\left(y_{0}^{\mu_{2}}\right)\left(y_{0}^{\mu_{1}}-y_{0}^{\mu_{2}}\right)
$$

Thus,

$$
\begin{aligned}
& \mathscr{J}\left(\mu_{1}\right)-\mathscr{J}\left(\mu_{2}\right) \\
& \geq \mathbb{E}\left[\Psi_{y}\left(y_{0}^{\mu_{2}}\right)\left(y_{0}^{\mu_{1}}-y_{0}^{\mu_{2}}\right)\right] \\
&+\mathbb{E} \int_{0}^{T}\left[\int_{U} l^{q}(t, a) \mu_{1 t}(d a)\right. \\
&\left.\quad-\int_{U} l^{\mu}(t, a) \mu_{2 t}(d a)\right] d t .
\end{aligned}
$$

We remark from (47) that $Q_{0}^{\mu}=\Psi_{y}\left(y_{0}^{\mu}\right)$. Then, we have

$$
\begin{aligned}
& \mathscr{J}\left(\mu_{1}\right)-\mathscr{J}\left(\mu_{2}\right) \\
& \geq \mathbb{E}\left[\Psi_{y}\left(y_{0}^{\mu_{2}}\right)\left(y_{0}^{\mu_{1}}-y_{0}^{\mu_{2}}\right)\right] \\
& +\mathbb{E} \int_{0}^{T}\left[\int_{U} l^{q}(t, a) \mu_{1 t}(d a)\right. \\
& \left.-\int_{U} l^{\mu}(t, a) \mu_{2 t}(d a)\right] d t .
\end{aligned}
$$

Thus,

$$
\begin{aligned}
& \mathscr{J}\left(\mu_{1}\right)-\mathscr{J}\left(\mu_{2}\right) \\
& \geq \mathbb{E}\left[Q_{0}^{\mu_{2}}\left(y_{0}^{\mu_{1}}-y_{0}^{\mu_{2}}\right)\right] \\
&+\mathbb{E} \int_{0}^{T}\left[\int_{U} l^{q}(t, a) \mu_{1 t}(d a)\right. \\
&\left.\quad-\int_{U} l^{\mu}(t, a) \mu_{2 t}(d a)\right] d t .
\end{aligned}
$$


By applying Itô’s formula to $Q_{t}^{\mu}\left(y_{t}^{q}-y_{t}^{\mu}\right)$, we obtain

$$
\begin{aligned}
& \mathbb{E}\left[Q_{0}^{\mu_{2}}\left(y_{0}^{\mu_{1}}-y_{0}^{\mu_{2}}\right)\right] \\
& =\mathbb{E}\left[Q_{T}^{\mu_{2}}\left(y_{T}^{\mu_{1}}-y_{T}^{\mu_{2}}\right)\right] \\
& -\mathbb{E} \int_{0}^{T} \mathscr{H}_{y}\left(t, y_{t}^{\mu_{2}}, z_{t}^{\mu_{2}}, \mu, Q_{t}^{\mu_{2}}, R_{t}^{\mu_{2}}\right) \\
& \quad \times\left(y_{t}^{\mu_{1}}-y_{t}^{\mu_{2}}\right) d t \\
& -\mathbb{E} \int_{0}^{T} \mathscr{H}_{z}\left(t, y_{t}^{\mu_{2}}, z_{t}^{\mu_{2}}, \mu_{2 t}, Q_{t}^{\mu_{2}}, R_{t}^{\mu_{2}}\right) \\
& \quad \times\left(z_{t}^{\mu_{1}}-z_{t}^{\mu_{2}}\right) d t \\
& +\mathbb{E} \int_{0}^{T} Q_{t}^{\mu_{2}}\left[\int_{U} f^{q}(t, a) \mu_{1 t}(d a)\right. \\
& \left.\quad-\int_{U} f^{\mu}(t, a) \mu_{2 t}(d a)\right] d t \\
& +\mathbb{E} \int_{0}^{T} R_{t}^{\mu_{2}}\left[\int_{U} g^{q}(t, a) \mu_{1 t}(d a)\right. \\
& \left.-\int_{U} g^{\mu}(t, a) \mu_{2 t}(d a)\right] d t .
\end{aligned}
$$

Then,

$$
\begin{gathered}
\mathscr{J}\left(\mu_{1}\right)-\mathscr{J}\left(\mu_{2}\right) \\
\geq \mathbb{E} \int_{0}^{T}\left[\mathscr{H}\left(t, y_{t}^{\mu_{2}}, z_{t}^{\mu_{2}}, \mu_{1 t}, Q_{t}^{\mu_{2}}, R_{t}^{\mu_{2}}\right)\right. \\
\left.-\mathscr{H}\left(t, y_{t}^{\mu_{2}}, z_{t}^{\mu_{2}}, \mu_{2 t}, Q_{t}^{\mu_{2}}, R_{t}^{\mu_{2}}\right)\right] d t \\
-\mathbb{E} \int_{0}^{T} \mathscr{H}_{y}\left(t, y_{t}^{\mu_{2}}, z_{t}^{\mu_{2}}, \mu_{2 t}, Q_{t}^{\mu_{2}}, R_{t}^{\mu_{2}}\right) \\
\quad \times\left(y_{t}^{\mu_{1}}-y_{t}^{\mu_{2}}\right) d t \\
-\mathbb{E} \int_{0}^{T} \mathscr{H}_{z}\left(t, y_{t}^{\mu_{2}}, z_{t}^{\mu_{2}}, \mu_{2 t}, Q_{t}^{\mu_{2}}, R_{t}^{\mu_{2}}\right) \\
\times\left(z_{t}^{\mu_{1}}-z_{t}^{\mu_{2}}\right) d t .
\end{gathered}
$$

Since $\mathscr{H}$ is convex in $(y, z)$ and linear in $\mu$, then by using the Clarke generalized gradient of $\mathscr{H}$ evaluated at $\left(y_{t}, z_{t}, \mu\right)$ and the necessary optimality conditions, that

$$
\begin{aligned}
\mathscr{H}( & \left.t, y_{t}^{\mu_{1}}, z_{t}^{\mu_{1}}, \mu_{1 t}, Q_{t}^{\mu_{1}}, R_{t}^{\mu 1}\right) \\
& -\mathscr{H}\left(t, y_{t}^{\mu_{2}}, z_{t}^{\mu_{2}}, \mu_{2 t}, p_{t}^{\mu_{2}}, Q_{t}^{\mu_{2}}, R_{t}^{\mu_{2}}\right) \\
\geq & \mathscr{H}_{y}\left(t, y_{t}^{\mu_{2}}, z_{t}^{\mu_{2}}, \mu_{2 t}, Q_{t}^{\mu_{2}}, R_{t}^{\mu_{2}}\right)\left(y_{t}^{\mu_{1}}-y_{t}^{\mu_{2}}\right) \\
& \quad+\mathscr{H}_{z}\left(t, y_{t}^{\mu_{2}}, z_{t}^{\mu_{2}}, \mu_{2 t}, Q_{t}^{\mu_{2}}, R_{t}^{\mu_{2}}\right)\left(z_{t}^{\mu_{1}}-z_{t}^{\mu_{2}}\right)
\end{aligned}
$$

or equivalently

$$
\begin{aligned}
0 \leq & \mathscr{H}\left(t, y_{t}^{\mu_{1}}, z_{t}^{\mu_{1}}, \mu_{1 t}, Q_{t}^{\mu_{1}}, R_{t}^{\mu 1}\right) \\
& -\mathscr{H}\left(t, y_{t}^{\mu_{2}}, z_{t}^{\mu_{2}}, \mu_{2 t}, Q_{t}^{\mu_{2}}, R_{t}^{\mu_{2}}\right) \\
& -\mathscr{H}_{y}\left(t, y_{t}^{\mu_{2}}, z_{t}^{\mu_{2}}, \mu_{2 t}, Q_{t}^{\mu_{2}}, R_{t}^{\mu_{2}}\right)\left(y_{t}^{\mu_{1}}-y_{t}^{\mu_{2}}\right) \\
& -\mathscr{H}_{z}\left(t, y_{t}^{\mu_{2}}, z_{t}^{\mu_{2}}, \mu_{2 t}, Q_{t}^{\mu_{2}}, R_{t}^{\mu_{2}}\right)\left(z_{t}^{\mu_{1}}-z_{t}^{\mu_{2}}\right) .
\end{aligned}
$$

Then, from (60), we get

$$
\mathscr{J}\left(\mu_{1}\right)-\mathscr{J}\left(\mu_{2}\right) \geq 0 .
$$

The theorem is proved.

\section{Conflict of Interests}

The author declares that there is no conflict of interests regarding the publication of this paper.

\section{Acknowledgment}

This work is partially supported by The Algerian PNR Project no. $8 / \mathrm{u} 07 / 857$

\section{References}

[1] E. Pardoux and S. G. Peng, "Backward doubly stochastic differential equations and systems of quasilinear SPDEs," Probability Theory and Related Fields, vol. 98, no. 2, pp. 209-227, 1994.

[2] Y. Shi, Y. Gu, and K. Liu, "Comparison theorems of backward doubly stochastic differential equations and applications," Stochastic Analysis and Applications, vol. 23, no. 1, pp. 97-110, 2005.

[3] M. N’zi and J. M. Owo, "Backward doubly stochastic differential equations with discontinuous coefficients," Statistics \& Probability Letters, vol. 79, no. 7, pp. 920-926, 2009.

[4] S. Peng and Y. A. Shi, "A type of time-symmetric forwardbackward stochastic differential equations," Comptes Rendus Mathématique. Académie des Sciences. Paris, vol. 336, no. 9, pp. 773-778, 2003.

[5] M. N'zi and J.-M. Owo, "Backward doubly stochastic differential equations with non-Lipschitz coefficients," Random Operators and Stochastic Equations, vol. 16, no. 4, pp. 307-324, 2008.

[6] S. Bahlali, "Necessary and sufficient optimality conditions for relaxed and strict control problems," SIAM Journal on Control and Optimization, vol. 47, no. 4, pp. 2078-2095, 2008.

[7] S. Bahlali and A. Chala, "A general optimality conditions for stochastic control problems of jump diffusions," Applied Mathematics and Optimization, vol. 65, no. 1, pp. 15-29, 2012.

[8] A. Chala, "The relaxed optimal control problem of forwardbackward stochastic doubly systems with Poisson jumps and its application to LQ problem," Random Operators and Stochastic Equations, vol. 20, no. 3, pp. 255-282, 2012.

[9] B. Mezerdi and S. Bahlali, "Necessary conditions for optimality in relaxed stochastic control problems," Stochastics and Stochastics Reports, vol. 73, no. 3-4, pp. 201-218, 2002. 
[10] S. Bahlali and B. Gherbal, "Optimality conditions of controlled backward doubly stochastic differential equations," Random Operators and Stochastic Equations, vol. 18, no. 3, pp. 247-265, 2010.

[11] Y. Han, S. Peng, and Z. Wu, "Maximum principle for backward doubly stochastic control systems with applications," SIAM Journal on Control and Optimization, vol. 48, no. 7, pp. 42244241, 2010. 


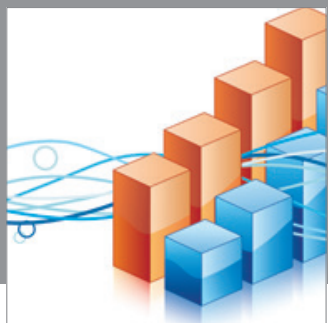

Advances in

Operations Research

mansans

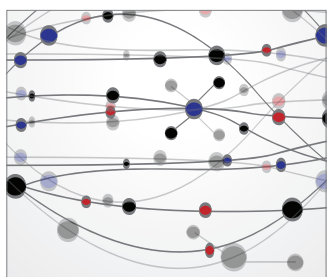

The Scientific World Journal
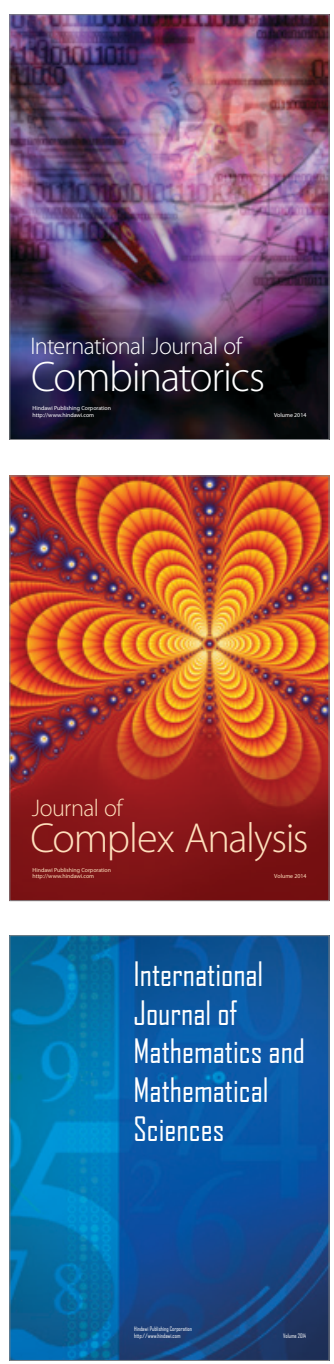
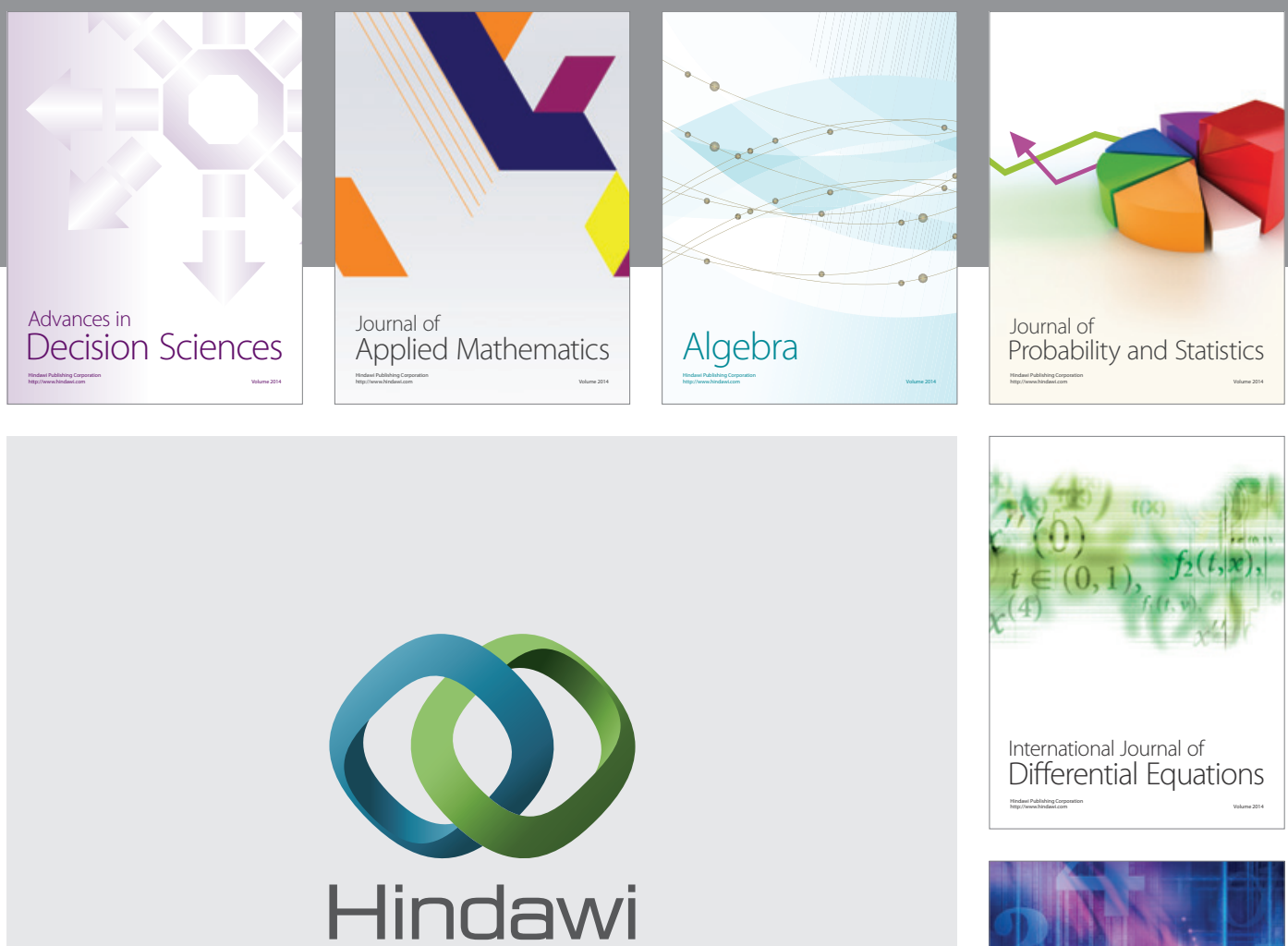

Submit your manuscripts at http://www.hindawi.com
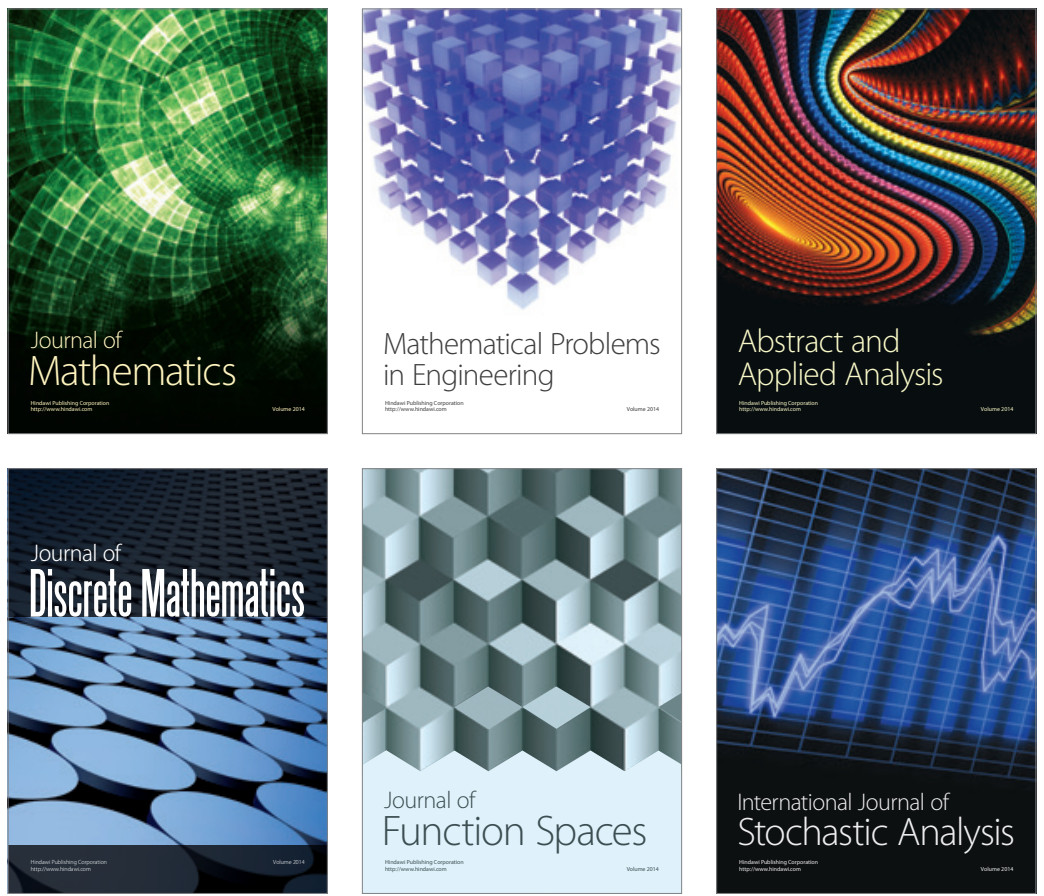

Journal of

Function Spaces

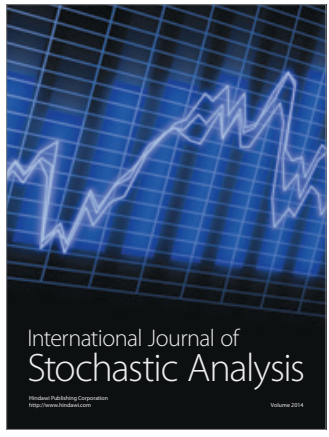

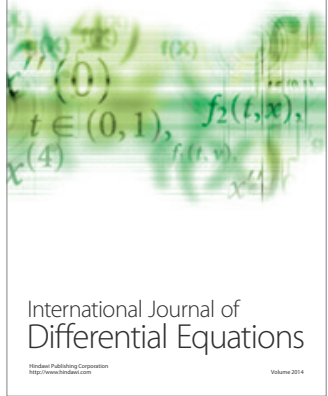
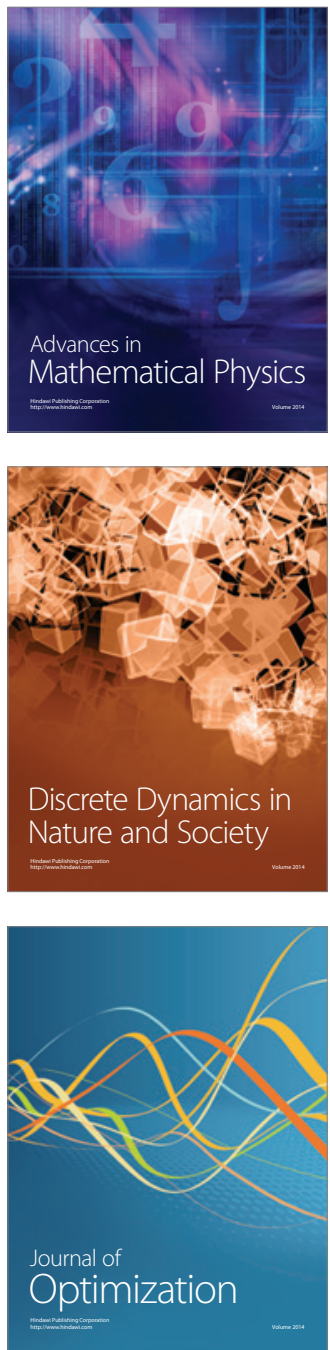